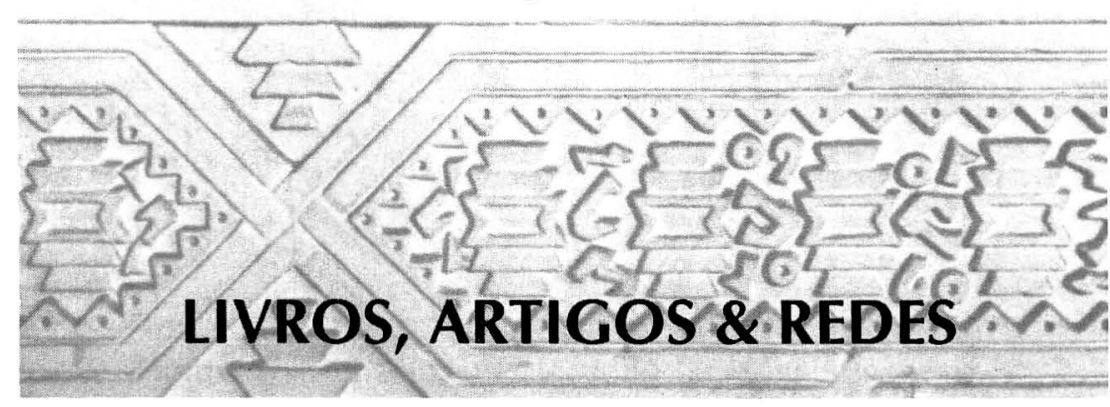

\section{INICIATIVA DE LA ORGANIZACIÓN PANAMERICANA DE LA SALUD EN HISTORIA DE SALUD PÚBLICA}

Hace varios años que la OPS ha tenido el interés en traer el enfoque de las ciencias sociales hacia la salud pública, y como parte cle este interés, en los últimos años varios projectos de investigación en historia de salud pública en América Latina y el Caribe han sido financiados por la OPS. Con este creciente interés en historia, el Programa de Investigación y Desarrollo Tecnológico en Salud de la División de Salud y Desarrollo de la OPS ha creado una iniciativa de historia con el fin de apoyar el campo de investigación en historia de salud pública en la región.

Hay que destacar desde el inicio que el objetivo de la iniciativa no es de promover historia hecha necesariamente por salubristas sino cle fortalecer la investigación en el campo de historia de salud pública, así despertando el interés de los propios historiadores. Con este fin hemos trabajado en una iniciativa con cuatro líneas de trabajo:

1. una red de investigadores

2. una bibliografia de historia cle salud pública

3. un concurso de investigación

4. una base de syllabus para apoyar a la enseñanza en historia de salud pública

1. Se espera que la Red facilite las comunicaciones y colaboraciones entre los investigadores de distintos países y sirva como un boletín continuo de las actividades, nuevos desarrollos y preocupaciones del campo. Los usos de la Red incluyen el intercambio de temas, métodos de investigación y enseñanza, nuevas tendencias en los campos de historia e historia de ciencias, y la creación de un espacio para debates intelectuales entre los usuarios. En la Red se puede hacer anuncios sobre congresos, jornadas, puestos de trabajo, becas, subvenciones, y también se puede di- fundir información sobre nuevos libros, bases de datos, catálogos de archivos, colecciones de manuscritos, bibliografías, o cualquier otra información que uno estime relevante. La belleza de la Red es que es descentralizada y cada uno de los usuarios puede alimentarla, enviando un mensaje por el correo electrónico. La Red está abierta a todos los interesados, sin límite. Hasta la fecha más de 140 personas han sido invitadas.

2. La creación de una bibliografia de historia de salud pública surge de una inquietud de que aunque existen muchos trabajos sobre la historia de salud pública en la región, hay poco conocimento de estos trabajos de país a país porque son publicados en revistas y libros con poca difusión o quedan en forma de literatura gris (especialmente tesis de maestría y doctorado).

Además una bibliografía secunclaria es imprescindible para la mejoría de la calidad de la investigación actual. La bibliografía dará más visibilidad a estudios cle la región a través de la difusión internacional de la bibliografía y espero que nos mandarán copias de sus trabajos para que sean incorporados.

El otro aspecto innovador de la bibliografía es su forma de reproduccíon. En vez de publicar la bibliografía en un libro, hemos creado una base de datos en Microisis, el programa gratis de la Unesco, y será enviado a todos los usuarios interesados en forma de disquete (incluyendo las referencias y una forma compacta de Microisis) para que acceden facilmente a la bibliografía en sus casas y instituciones. Este esfuerzo no solo representa un gran ahorro de dinero, sino provee la oportunidad de actualizar la bibliografía cada año, así aumentando su utilidad a los investigadores. Pagando solo para un disquete, cada usuario recibirá una versión actualizada de la bibliografía. Finalmente, la bibliografía estará institucionalizada en un centro de investigación latinoamericano, el cual tendrá la 
responsabilidad cle fotocopiar trabajos de la bibliografía para responder a pedidos de usuarios (los cual pagarán un costo mínimo para recibir los trabajos). Creemos que este servicio será de gran utilidad dada la gran dificultad de obtener trabajos en muchos países. Hasta la hora hemos agregado más de mil referencias, con más de 70 sobre historia de la enfermería en salud pública. Ojalá que a través de la red, ustedes nos informarán de nuevas referencias y de como perfeccionar la bibligrafía.

3. Como parte de la misión de la OPS de mejorar las condiciones de salud de la región, provee subvenciones para investigaciones con este fin que son evaluadas por un comité asesor cuatro veces al año. Además, periódicamente la OPS lanza convocatorias para concursos de investigación en áreas de interés especial. Con esta intencionalidad hemos desiñado un concurso de investigación bajo el tema 'Reforma Sanitaria en Perspectiva Historica' el cual esperamos lanzar a principios de 1995, otorgando tres subvenciones en el primer año. El concurso clefine reforma sanitaria de manera muy amplia, incluyendo los siguientes temas: la emergencia del estado benefactor y los sistemas nacionales de salud; el papel de diversos actores como gremios, sindicatos, y movimientos populares; las ideologías de salud en distintas épocas; el impacto de las influencias europeas y norteamericanas en las reformas; la clefinición de prioridades en las políticas de salud; la relación entre las reformas y la enseñanza de la salud pública; el papel de la medicina tradicional; y el rol que ha jugado el medio ambiente en la reforma sanitaria, entre otros temas.

4. La cuarta y última línea de trabajo por el momento es el apoyo a la enseñanza en la historia de salud pública de la región en escuelas de salud pública y enfermería tanto como en facultades de medicina y historia. Estamos agregando una base de syllabus (marcos o contenidos de cursos) para redis- tribuir a docentes que están renovando sus cursos o creando nuevos cursos en el área cle historia de salud.

Hay que hacer hincapié en que estas líneas cle trabajo no son inclependientes sino complementarias. Es decir que la Red sirve de apoyo a los syllabus y la bibliografía; la bibliografía es un aporte a la investigación en la preparación de propuestas y trabajos para el concurso; el apoyo a la enseñanza sirve para despertar más interés en la Red y la investigación etc. Esperamos realmente que esta iniciativa pueda fortalecer el campo de historia de salud pública en América Latina y el Caribe y que habrá un gran intercambio de ideas y actividades entre los miembros de la Red desde ahora en adelante. Su participación a nivel cle comentarios y sugerencias, junto con la propia investigacion son un aporte imprescinclible en el desarrollo de esta iniciativa.

La suscripción a la Red se hacer enviando sus direcciones electrónicas, escribiendo

<subscribe HISALP-L su nombre>

a: listserv@db2.nlm.nih.gov.

Después se siguen las instrucciones indicadas en la respuesta del server. Para mayor información sobre la Red o las otras líneas cle trabajo, por favor comuníquense con:
Anne-Emanuelle Birn
tel.: (202) 861-3234
fax: (202) 861-8472
e-mail: birnanne@paho.org
La dirección tradicional es:
HDP/HDR, 525
$23^{\text {rd }}$ St. NW, Washington-DC 20037, EUA.

Bienvenidos a la Red.

Anne Emanuelle Birn Consultora do Programa de Investigação e Desenvolvimento Tecnológico em Saúcle da Organização Panamericana de Saúde. 